\title{
Movilidad residencial regional, migraciones y balance territorial en la Comunidad de Madrid durante la crisis económica*
}

\author{
Julio A. del-Pino-Artacho \\ UNED. Departamento Sociología I (Teoría, Metodología y Cambio Social) \\ jadelpino@poli.uned.es
}

\section{Resumen}

En la actualidad, el balance regional interno de la Comunidad de Madrid depende fundamentalmente de las corrientes de movilidad. Este trabajo analiza la movilidad residencial regional y las migraciones en relación con su efecto sobre los procesos de concentración o desconcentración de la población. Para ello, enfocamos nuestra atención en la evolución de la movilidad a lo largo de la crisis económica y en las diferencias de este fenómeno entre nativos y no nativos. Utilizamos datos de diversas fuentes (censos, Padrón y Estadística de Variaciones Residenciales) para tres áreas (capital, área metropolitana y periferia) y definimos cuatro tipos de procesos de desplazamiento: centralización, metropolización, descentralización periférica y movilidad interna. Los resultados señalan que, durante la crisis, la reducción del volumen de movilidad no ha supuesto ninguna reversión de los procesos iniciados a finales de los años noventa. Tienden a reducirse los efectos temporales de la inmigración extranjera ("falsa» centralización, distribución), mientras que la tendencia a la desconcentración metropolitana entra en disputa con una creciente corriente de centralización. La movilidad regional y migratoria interna de la población no nativa asentada comienza a converger con la de los nativos.

Palabras clave: movilidad residencial; Comunidad de Madrid; migraciones; crisis económica

* El presente trabajo se ha beneficiado de la financiación otorgada por la Red de Excelencia de Investigaciones Socioterritoriales y Desarrollo Rural (CSO2014-52862-REDT).

El autor desea expresar su agradecimiento a los evaluadores anónimos, que han orientado una mejora sustancial del estudio llevado a cabo. 
Abstract. Regional residential mobility, migrations and spatial balance in the Community of Madrid during the economic crisis

At present, the internal regional balance of the Community of Madrid basically depends on flows of mobility. This paper analyzes regional residential mobility and migrations as related to processes of population concentration and deconcentration. To this end, we focus our attention on the evolution of mobility throughout the economic crisis and on the differences in mobility between native and non-native populations. Population and mobility data from various sources (Census, Register and Residential Variation Statistics) are used for three areas (the central city, the metropolitan belt and the peripheral areas), and four types of mobility processes are defined: centralization, metropolization, peripheral decentralization and internal mobility. The results indicate that, during the crisis, the reduction in the volume of mobility did not mean a reversal of the increasing mobility initiated at the end of the 1990s. Temporary effects of foreign immigration ("false» centralization, distribution) tend to be reduced, while the tendency towards metropolitan deconcentration become in dispute with a growing trend of centralization. Mobility of stablished non-native population begins to converge with the mobility of the native population.

Keywords: residential mobility; Community of Madrid; migrations; economic crisis

\section{Sumario}

1. Planteamiento

2. Metodología

3. Evolución demográfica regional

4. Variaciones residenciales de nativos y no nativos durante la crisis económica
5. Movilidad y reestructuración territorial de la región madrileña

6. Conclusión

Referencias bibliográficas

\section{Planteamiento}

La distribución de la población en la región urbana madrileña ha sufrido cambios sustanciales durante los últimos veinte años. En un escenario de tenue crecimiento vegetativo, los cambios demográficos desde la década de 1980 venían siendo determinados por corrientes de movilidad fundamentalmente protagonizadas por nacionales, con un gran peso de la dinámica de movilidad interna de la región. Desde finales de la década de 1990, se ha pasado a un escenario más complejo en el que, a la intensificación general de la movilidad, se une la explosión de nuevas corrientes migratorias de alcance internacional. En este trabajo, tratamos de comprender la relación entre estos cambios y la organización socioespacial de la región.

La observación de la reorganización del espacio se va a centrar en los balances de movilidad residencial entre las diferentes áreas que conforman la región y entre cada una de ellas y el exterior de la región. Se entiende que este es uno de los mecanismos principales que regula la relación demográfica entre las diferentes zonas de la Comunidad. Sin embargo, esta movilidad ha de entenderse 
dentro de un marco general dominado por corrientes globalizadoras, a la vez que generador de estrategias de acción particulares en el territorio, que tratan de absorber los cambios en clave de adaptación.

Vivimos en una era de las movilidades (Urry, 2000), donde buena parte de las relaciones han dejado de estar ancladas en lugares cercanos para atravesar el territorio, bien a través de los transportes, bien a través de las comunicaciones, lo que ha ensanchado el espacio donde se desenvuelven las relaciones cotidianas y, también, el conjunto de las posibles relaciones que podemos entablar durante toda la vida (Giddens, 1985). En este escenario de compresión espaciotemporal (Harvey, 1989), la movilidad se convierte en una forma de capital fundamental, la motilidad o capacidad para moverse en un determinado sistema de movilidad (Kaufmann et al., 2004). Desde esta perspectiva, todo tipo de movilidad (residencial, cotidiana, migratoria, turística, etc.) se relaciona con la propia estructura social, al nivel de otros factores como la propiedad, la renta o la educación. Aquellos grupos que más recursos tienen, o que mejor los utilizan, para moverse adquieren ventajas duraderas y significativas sobre otros grupos.

En sentido macro, la era de la movilidad está gobernada por las fuerzas de la globalización, como «conjunto de procesos espaciotemporales de cambio que apuntalan una transformación de los asuntos humanos, mediante el crecimiento de sus relaciones y la expansión de las actividades humanas a través de los continentes y regiones» (Held et al., 1999). En sentido micro, el manejo de las movilidades se realiza a través de estrategias que los actores utilizan para adaptar su situación estructural a las cambiantes circunstancias históricas que viven (Holling, 2001). Otros mecanismos de adaptación, como pueden ser el tamaño y la organización de los hogares o la acumulación de capital educativo, conviven con las estrategias de adaptación en relación con la movilidad (Folke, 2006). Así pues, la movilidad es una poderosa herramienta que los actores (individuos o colectivos) utilizan para reaccionar en contextos de cambio (Locke et al., 2000). Desde este punto de vista relacional, el territorio se convierte en el resultado no intencionado de la aplicación de las diversas pautas que animan las prácticas de movilidad de los diversos grupos que transitan (Sheller y Urry, 2016).

La translación de este marco a la realidad de la organización socioespacial de Madrid nos permite observar la región madrileña bajo el influjo de fuerzas globalizadoras y sistemas de acción locales, con anclajes históricos bien definidos. En términos teóricos, la lógica sistémica nacional que alimentaba a Madrid como capital de España va dando paso a una lógica más reticular y globalizada. Por un lado, el principio jerárquico que animaba el sistema nacional de asentamientos, por el que Madrid concentraba las funciones dominantes del sistema urbano estatal, tiende a reducir su fuerza, con procesos como el crecimiento de las ciudades medias. Al mismo tiempo, las jerarquías urbanas globales se imponen, otorgando a Madrid, como a otras ciudades del sur de Europa, como Barcelona o Milán, un papel de ciudad global regional. Por otra parte, surgen nuevas relaciones urbanas en red, que conectan lugares de diverso tamaño a 
través de relaciones funcionales que desbordan la lógica puramente territorial (Del Pino, 2001). En términos demográficos, se pasa de las migraciones laborales nacionales de sentido único (en las que los migrantes comenzaban una nueva vida fuera sin expectativa cierta de regreso) a las movilidades circulares (Newland, 2009) y las migraciones transnacionales (Waldinger, 2013). En este escenario, la movilidad, tanto en el interior de la región como en relación con el exterior, se convierte en un factor clave que permite la adaptación dinámica de poblaciones y hogares a contextos cambiantes, como tendremos la oportunidad de observar en relación con la crisis económica.

Así pues, Madrid bascula entre tres grandes lógicas, que combinan el alcance de las relaciones (estatal o global) y el carácter más bien sistémico o reticular de las mismas. Por una parte, Madrid actúa como cabeza de un sistema urbano nacional (capital, sede del Gobierno y de las instituciones del Estado). Por otro lado, actúa como ciudad global regional (Sassen, 2000), acogiendo sedes de grandes corporaciones globales o promocionando un hub aeroportuario regional. Por último, Madrid funciona como un nodo dentro de la red urbana global (Castells, 1997), compitiendo con otros muchos lugares por atraer flujos de bienes y servicios (polos tecnológicos, eventos culturales, torneos deportivos, grandes inversiones, etc.).

Estos mismos vectores resultan esclarecedores a la hora de contemplar los procesos de configuración interna de la propia región. De un lado, la capital actúa como un potente centro de gravedad, cabeza del sistema urbano local y catalizador de las relaciones de la región con el entorno global. En este sentido, buena parte del proceso de metropolización se constituye como un mero desbordamiento de la ciudad central, lo que da lugar a espacios residenciales, industriales y de servicios dependientes del centro urbano. Ello se observa preferentemente con el crecimiento de los municipios adyacentes del sur y el este metropolitano.

Sin embargo, esta fuerza centrípeta, centralizadora, convive desde hace décadas con procesos descentralizadores, centrífugos, que no solo alimentan el alejamiento físico del centro y la adopción de entornos menos densos, sino que también vienen unidos al desarrollo de cierta autonomía de los núcleos no centrales y la creación de interdependencias regionales menos jerarquizadas (Moreno, 1999; Vinuesa, 2004). Esta descentralización adopta, como se ha señalado frecuentemente, la forma de la ciudad dispersa, con alta especialización residencial y uso extensivo del suelo (Gutiérrez y García-Palomares, 2007), lo que da lugar a serios problemas de sostenibilidad, como los derivados del transporte (Monzón y De la Hoz, 2009). Pero también, en consonancia con la idea de la constitución de una red de nodos urbanos, esta descentralización conlleva la formación de una región metropolitana (Valenzuela, 2011), donde la dotación de servicios y la diversificación funcional promueve las bases para el desarrollo de estructuras urbanas interdependientes de carácter policéntrico (Gallo y Garrido, 2012; Gallo et al., 2010; Roca et al., 2011), que eventualmente conectan subcentros del sistema regional con la red urbana global (a través de polos tecnológicos, infraestructuras o dotaciones singulares). 
A pesar de la objetividad aparente de los procesos de centralización y descentralización, estos no resultan nada fáciles de evaluar. Por ejemplo, el fuerte peso específico de la ciudad central en la configuración regional parece puesto en entredicho al observar su decreciente población durante las últimas décadas (Galiana y Vinuesa, 2012). En sentido contrario, un proceso descentralizador como el crecimiento de la ciudad dispersa, siguiendo el modelo norteamericano de especialización residencial y gran consumo de espacios, engorda paradójicamente la centralidad de Madrid, con cuya capital la ciudad dispersa mantiene relaciones de estricta dependencia funcional. De este modo, la adopción de formas descentralizadas por desbordamiento de la capital, como las ciudades dormitorio, y la constitución del área metropolitana no siempre provoca diversidad, sino una configuración regionalizada de la ciudad, que permanece hasta hoy (Roca et al., 2011).

La originalidad de la presente investigación consiste en observar los indicadores de movilidad como expresión a la vez de las corrientes de reestructuración global y de las estrategias de los actores en el territorio, que se producen en un contexto espaciotemporal concreto, marcado por el episodio de inmigración internacional iniciado a finales de la década de 1990 y por la gran recesión económica de finales de la década de 2000. De este modo, relacionamos la reestructuración territorial con el origen de los migrantes y estudiamos los efectos de tendencia y coyunturales que la movilidad residencial y las migraciones tienen sobre el territorio.

La movilidad residencial y las migraciones de los no nativos resulta especialmente ilustrativa de estos procesos. Los extranjeros llegados desde finales de los años noventa encarnan el condicionante global de las movilidades que les ha hecho viajar miles de kilómetros por razones económicas, a la vez que podría hacerles más volátiles, al permitir la reversibilidad de los movimientos ante un escenario cambiante. Además, se ven especialmente expuestos a utilizar estrategias de movilidad residencial, tanto por su estructura demográfica, tendente a concentrar procesos de formación de hogares o reagrupamientos, como por sus condiciones de asentamiento (a menudo, por pasos, transitando por diversos domicilios durante los primeros años) y por su menor arraigo territorial (especialmente, en términos de tenencia, pues utilizan mucho más el alquiler). Todos estos factores, tanto los relacionados con la llegada masiva de extranjeros como con su itinerario de asentamiento y/o su abandono eventual, tienen necesariamente consecuencias sobre los balances territoriales internos de la región (Recaño, 2016; Pozo y García-Palomares, 2011; Módenes, 2007; Bayona y Pujadas, 2014).

La focalización en el periodo de crisis no pretende la medición del impacto del mismo sobre la movilidad residencial. Más bien se sirve de ella para, por una parte, evaluar los efectos de tendencia que venían observándose y que la crisis solo es capaz de atemperar, como el incremento general de la movilidad o las tendencias hacia la desconcentración (Gil-Alonso et al., 2013). Por otra parte, el escenario de crisis invita a observar las estrategias de movilidad o de permanencia como formas de adaptación a un entorno claramente afectado por 
la disminución del empleo. La combinación del perfil no nativo, especialmente proclive a la movilidad, y el escenario de crisis plantea un objeto de extraordinario interés para comprender la utilización de la movilidad residencial como estrategia de adaptación de los hogares (López y Pérez, 2015; Gil-Alonso et al., 2015; Viruela, 2016).

El objetivo del trabajo es describir la dinámica de las pautas de movilidad que afectan a la configuración territorial interna de la Comunidad de Madrid en términos de centralización y descentralización, con especial atención al periodo de crisis económica.

Se trata de repasar, en primer lugar, la configuración histórica de Madrid, señalando sus principales características, particularmente el peso del crecimiento migratorio en la distribución de la población. En segundo lugar, se busca evidenciar el peso que la movilidad residencial y las migraciones adquieren en la región durante los últimos lustros, incluyendo el periodo de cambio que supone la crisis económica, de acuerdo con la idea de ascenso de la movilidad en las sociedades contemporáneas. Finalmente, se persigue conocer la dinámica de cada una de las corrientes de movilidad que configuran el espacio madrileño en términos de concentración y desconcentración.

Tras este planteamiento, se aborda la metodología de análisis y se realiza una aproximación histórica al caso de Madrid. A continuación, se analizan los datos de movilidad residencial a la luz de la gran recesión, incidiendo en el origen nativo o no de los protagonistas. Finalmente, se analizan las consecuencias para el territorio, en términos de concentración y desconcentración, de la movilidad residencial a lo largo del periodo, planteando algunas líneas de futura indagación.

\section{Metodología}

Vamos a centrar nuestras observaciones en la movilidad residencial regional y en las migraciones, como indicadores principales de la movilidad que configura el territorio. Sin embargo, las variaciones residenciales son solo indicativas de procesos de movilidad más amplios (Del Pino, 2015) que incluyen otras movilidades, como el commuting, el turismo o la segunda residencia.

El escenario de los movimientos es la Comunidad de Madrid, dividida a efectos meramente heurísticos en tres grandes regiones funcionales: la capital, el área metropolitana (AM) y la periferia. Desde la etapa modernizadora hasta la actualidad, diversos estudios han puesto de manifiesto especialmente el desbordamiento de Madrid como área funcional más allá de estos límites (Salcedo, 1977). Otros trabajos han incorporado particularmente la cuestión del commuting para una mejor delimitación de las áreas urbanas en España (Feria, 2008; Roca et al., 2011). Sin embargo, el carácter histórico y administrativo de la división provincial, así como la posibilidad de compararlo con otras comunidades o provincias, permiten el uso de la presente división con provecho. La delimitación (mapa 1) se basa en la clasificación estadística europea (NUT 4), realizando agregaciones de carácter funcional. Se delimita como un área 
Mapas 1. Capital, área metropolitana y periferia

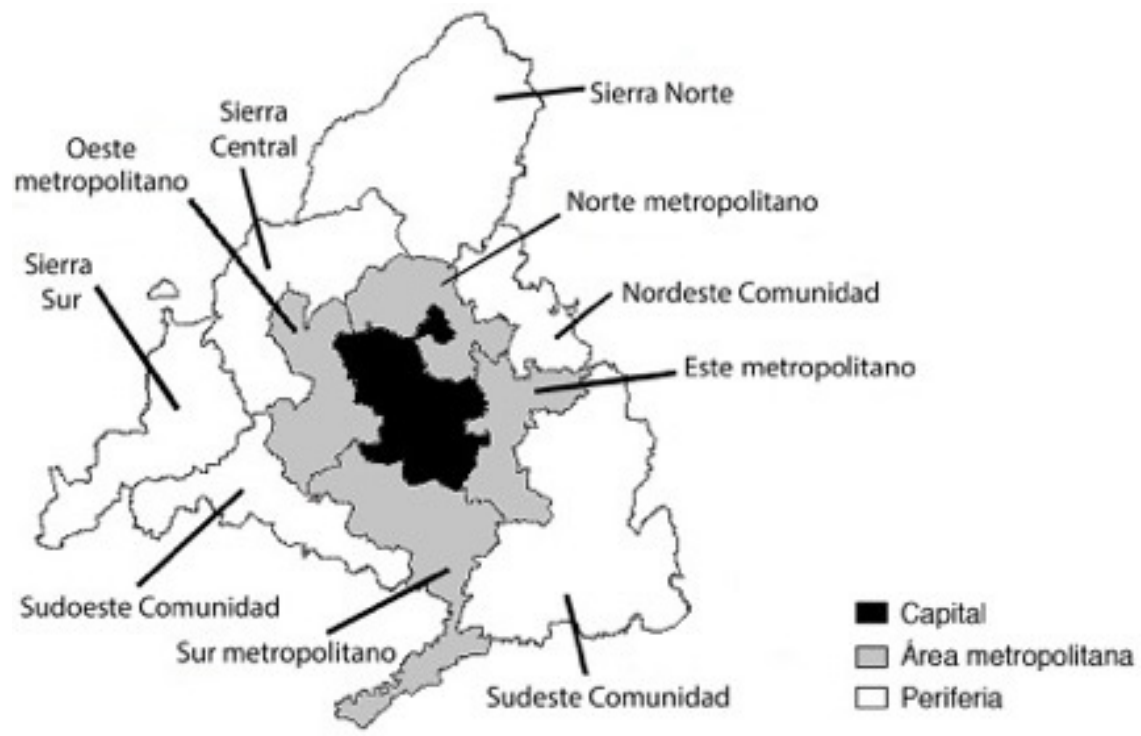

Fuente: elaboración propia.

separada al municipio de la capital. El AM está constituida por el conjunto de los cuarenta municipios que conforman las cuatro áreas estadísticas que rodean la capital (norte, sur, este y oeste). Por último, la periferia está constituida por el conjunto del resto de áreas, en una situación más alejada del centro: las zonas de sierra (Sierra Norte, Sierra Central y Sierra Sur) y el resto de zonas periféricas (el sudoeste, el sudeste y el nordeste de la Comunidad de Madrid).

Para el análisis de los datos, operamos en tres pasos encadenados. En primer lugar, analizamos la evolución demográfica histórica de la región madrileña. En segundo lugar, atendemos a la evolución más reciente de la movilidad residencial regional y las migraciones, haciendo hincapié en la distinción entre la movilidad de nativos y no nativos. Finalmente, observamos la dirección y el sentido de los movimientos, de modo que podamos relacionar las variaciones residenciales con los procesos de reestructuración en el territorio.

Todos los datos utilizados provienen de fuentes estadísticas producidas por el Instituto Nacional de Estadística (INE). En el análisis de la evolución demográfica, se han utilizado como indicadores fundamentales el número de habitantes y las tasas de crecimiento. En el caso del número de habitantes, se utilizan dos fuentes: para la evolución histórica (1900-2011), los censos y, para la evolución del periodo más reciente (1986-2015), año a año, el Padrón municipal. Obviamente, no se trata de armonizar las fuentes, sino de poder obtener medidas del proceso en dos escalas temporales bien diferentes. Para la presentación de las tasas de crecimiento vegetativo y migratorio, se ha procedido del 
siguiente modo. En primer lugar, se ha establecido el crecimiento total de la población $(\mathrm{CT})$ para cada año $(t)$, como la diferencia, en cada área $(i)$, entre la población (P) a 1 de enero del año $t+1$ y la población a 1 de enero del año $t$.

$$
C T_{\mathrm{i}}^{\mathrm{t}}=\mathrm{P}^{01-01-(\mathrm{t}+1)}-\mathrm{P}^{01-01-\mathrm{t}}
$$

Para las tasas de crecimiento vegetativo, se utilizan las cifras oficiales de crecimiento vegetativo del Movimiento Natural de la Población (MNP), de modo que se expresa como el saldo vegetativo (SV) de cada año en el ámbito de estudio por cada mil habitantes de ese ámbito a mitad del periodo.

$$
\mathrm{CV}_{\mathrm{i}}^{\mathrm{t}}=\frac{S \mathrm{~V}_{\mathrm{i}}^{\mathrm{t}}}{\mathrm{P}_{\mathrm{i}}^{\mathrm{t}}} \cdot 1000
$$

Las tasas de crecimiento migratorio se han hallado por el método inverso, es decir, restando al crecimiento interanual (CT) de la población las cifras de crecimiento vegetativo (SV). Este método implica que los valores de las tasas de crecimiento migratorio incluyen los errores de compatibilidad que pueda haber entre el crecimiento total y el crecimiento vegetativo. Este efecto suele ser mayor cuanto menor sea el tamaño de la población del área de estudio. Para el crecimiento migratorio, se ha calculado la diferencia entre el crecimiento total y el saldo vegetativo para cada año en cada ámbito y se ha puesto en relación con la población en ese ámbito, por cada mil habitantes.

$$
\mathrm{CM}_{\mathrm{i}}^{\mathrm{t}}=\frac{\mathrm{CT}_{\mathrm{i}}^{\mathrm{t}}-\mathrm{SV}_{\mathrm{i}}^{\mathrm{t}}}{\mathrm{P}_{\mathrm{i}}^{\mathrm{t}}} \cdot 1000
$$

Se ofrecen, finalmente, las tasas medias de crecimiento anual para periodos de cinco años, a partir de la suma de CV y CM. Para el periodo de crisis (20072015), se especifican las tasas de crecimiento anual.

El análisis de la movilidad reciente explota los datos de la Estadística de Variaciones Residenciales (EVR) en el periodo 1997-2015, con alusiones más específicas al periodo de crisis (2007-2015). Se escoge este periodo porque es en el año 2007 cuando la crisis económica comienza a notarse en los indicadores de desempleo, cuya tasa registra alzas trimestrales en la Comunidad de Madrid entre julio de 2007 y junio de 2010. El hecho de que las pautas de movilidad y migración registren este escenario de crisis con cierto retraso permite observar el cambio con mejor perspectiva.

Dada la importancia que tienen las trayectorias de los inmigrantes extranjeros en nuestro análisis, resulta importante señalar que se utiliza como criterio el origen o el lugar de nacimiento, no la nacionalidad, con lo que se despejan las complicaciones que los procesos de nacionalización conllevan para la comparación diacrónica de indicadores basados en la nacionalidad.

En cuanto a los tipos de variaciones residenciales registradas, se atiende a tres tipos de movimientos según su alcance: la movilidad residencial regional, compues- 
ta por aquellos movimientos intermunicipales dentro de la Comunidad de Madrid; las migraciones internas que se registran entre los municipios de la Comunidad de Madrid y otros municipios del resto de España, y las migraciones internacionales, entre municipios de la Comunidad de Madrid y países distintos de España.

La reorganización socioespacial a lo largo de las tres áreas funcionales definidas se persigue a través del cálculo de tasas de movilidad específicas que revelan los diversos procesos que se producen en el territorio. Para poder establecer comparaciones sobre el peso que tiene cada corriente en la configuración global del territorio de la Comunidad de Madrid, cada tasa utiliza como base su población total. Este criterio permite obtener un indicador comparable para cada corriente, que incorpora la distribución desigual de la población. De este modo se independiza de la importancia relativa que cada flujo tiene sobre la población del área de llegada, lo que ofrece una mejor perspectiva para pensar en la realidad de los procesos de concentración y desconcentración de la población en el conjunto de la Comunidad.

La movilidad residencial regional se ha dividido en cuatro tipos de movilidad, de acuerdo con su dirección y su sentido:

- Centralización: movimientos desde el AM y la periferia hasta la capital y movimientos desde la periferia hasta el AM.

- Metropolización: movimientos desde la capital hasta el AM.

- Desconcentración: movimientos desde la capital y el AM hacia la periferia.

- Internas: solo movilidad entre municipios dentro de la misma área (dentro de la capital, dentro del AM, dentro de la periferia).

Para el flujo de determinado tipo de movilidad (Fi), las tasas de movilidad (TM) en el año $t$ se calculan como la relación entre ese flujo y la población media anual $\left(\mathrm{P}^{\mathrm{t}}\right)$ de la Comunidad de Madrid por cada mil habitantes:

$$
\mathrm{TM}_{\mathrm{i}}^{\mathrm{t}}=\frac{\mathrm{F}_{\mathrm{i}}^{\mathrm{t}}}{\mathrm{P}^{\mathrm{t}}} \cdot 1000
$$

Las migraciones internas e internacionales se estudian separadamente para cada área a partir del saldo migratorio entre inmigraciones con origen en el exterior de la Comunidad (bien sea en otras provincias, en el caso de las migraciones internas, o en otros países, en el caso de las internacionales) y emigraciones con destino en el exterior de la Comunidad (a otras provincias o a otros países). Este procedimiento da lugar al cálculo de tasas de movilidad migratoria (TMM) para cada una de las dos modalidades migratorias $(i)$, interna o internacional, en cada área $(j)$, capital, AM y periferia, y cada año $(t)$. Las tasas ponen en relación el saldo migratorio de la modalidad en un área y año determinados con la población media de la Comunidad en ese año $\left(\mathrm{P}^{t}\right)$ por cada mil habitantes.

$$
\mathrm{TMM}_{\mathrm{ij}}^{\mathrm{t}}=\frac{S_{\mathrm{ij}}^{\mathrm{t}}}{\mathrm{P}^{\mathrm{t}}} \cdot 1000
$$




\section{Evolución demográfica regional}

Para 2015, la población empadronada de la Comunidad es de 6,4 millones de habitantes, casi la mitad de los cuales viven en la capital (3,1 millones), 2,8 millones en el AM y alrededor de medio millón de personas en la periferia. Madrid es una de las cabezas del sistema urbano español. Alberga casi un 14\% de la población residente en España, según los datos del Censo de 2011. La otra gran cabeza, Barcelona, alberga a 5,5 millones de habitantes y el 12\% de la población.

La evolución demográfica histórica de las tres áreas en las que se ha dividido la Comunidad de Madrid se formula en los términos clásicos del desarrollo de las ciudades modernas, con un proceso de centralización muy fuerte seguido de una progresiva desconcentración en diversas fases (Del Pino, 2013). Esta evolución (gráfico 1A) apunta al despegue de la ciudad central hasta 1930, seguido de cierta ralentización del crecimiento con ocasión de la Guerra Civil (1936-1939). Durante la postguerra, la ciudad comenzó a crecer intensamente, pero es a partir de 1950 y hasta 1980 cuando se produce el proceso de modernización alimentado por un fuerte éxodo rural que hará crecer la periferia (especialmente, la sur y este). Durante la fase de concentración en sentido estricto, la capital eleva su población en relación con el resto de la región, hasta llegar a concentrar a casi el 90\% de la población de la región en 1960. A partir de este momento, se produce una fase de desconcentración de la población en dos periodos. Durante las décadas de 1960 y 1970, las grandes migraciones desde las áreas rurales desbordan la ciudad y colonizan los pueblos de alrededor (algunos de los cuales ya habían sido agregados al municipio de la capital durante los años cuarenta y primeros años cincuenta). El proceso dominante es el de una metropolización que podría calificarse de densa, por las tipologías

Gráfico 1. Evolución histórica de la población. Número de habitantes (A) y distribución territorial (B) (1900-2011)

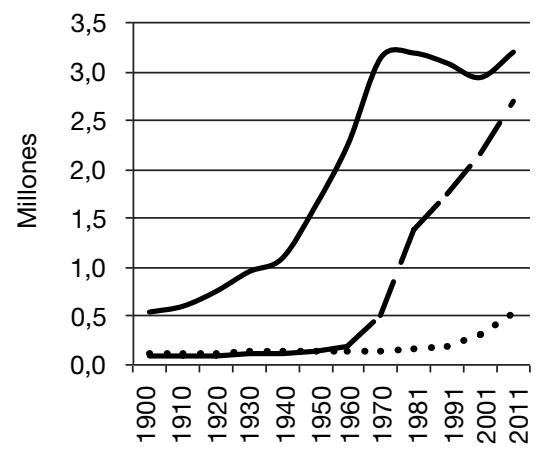

- Capital —-AM $\quad$-...Periferia

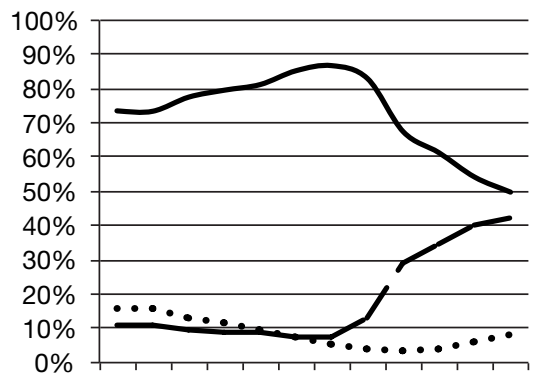

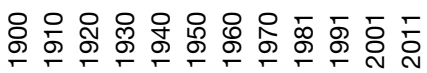

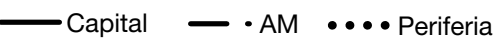

Fuente: Censos. INE. Elaboración propia. 
en altura en las que, poco a poco, se va asentar a los nuevos pobladores. Hacia 1980, comienza a observarse la salida de la capital de las nuevas clases medias, que alimentarán unas nuevas periferias, especialmente al oeste y al norte de la capital. Esta nueva fase de metropolización difusa (urban sprawl) se lleva a cabo en espacios menos densos relativamente cercanos a la ciudad, pero también, cada vez más, en lugares distantes, lo que da lugar a corrientes de entrada en áreas rurales de la periferia regional (Moreno, 1999; Vinuesa, 2004).

Con el nuevo siglo, la llegada de inmigrantes extranjeros volverá a transformar la población madrileña, que registrará un aumento muy significativo de la movilidad de toda clase, lo que provocará incluso el crecimiento de la población en la ciudad central, si bien, globalmente, este no afecta a los procesos de desconcentración en la región (Vinuesa, 2004).

Una caracterización más precisa de las diferentes áreas durante las últimas décadas la ofrecen las tasas de crecimiento anual correspondientes a cada una, desagregadas entre crecimiento vegetativo y crecimiento migratorio. Como vemos en el gráfico 2, según el carácter creciente o decreciente de las tasas, pueden observarse tres periodos. Entre 1986 y 1995, el escenario general es de desconcentración, con procesos de metropolización difusa, suburbanización y contraurbanización, que se encuentran alimentados por un descenso de la población de la capital. Durante el periodo de 1996 a 2005, el escenario se encuentra dominado por las altas tasas de inmigración protagonizadas por extranjeros. Por último, entre 2006 y 2015, nos encontramos ante el escenario de desaceleración del crecimiento demográfico que ha acompañado a la crisis económica.

En términos de composición, las tasas revelan que el crecimiento migratorio es más significativo que el natural para explicar las variaciones en las tasas de crecimiento total. Los cambios en el crecimiento natural están ligados íntima-

Gráfico 2. Tasas de crecimiento de la población por área, en tasas por mil según las medias anuales por lustro (1986-2015)
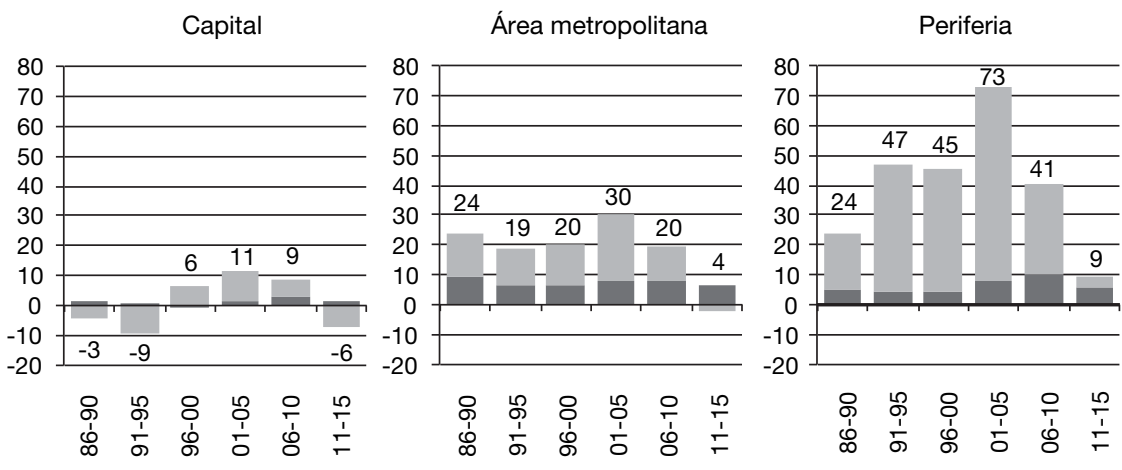

Tasa de crecimiento vegetativo

Tasa de crecimiento migratorio

Fuente: Padrón y MNP. Elaboración propia. La cifra junto a cada barra indica el crecimiento total. 
mente al migratorio, especialmente cuando los inmigrantes y los anfitriones difieren mucho en sus estructuras demográficas. En nuestro caso, las poblaciones autóctonas envejecidas, que apenas registran natalidad, contrastan mucho con las poblaciones jóvenes de inmigrantes laborales. Dada la contribución de la población inmigrante al crecimiento vegetativo, podemos decir que este resulta especialmente sensible a las decisiones sobre movilidad residencial que toman los extranjeros durante la crisis.

El análisis conjunto de las tasas de crecimiento (gráfico 2) y de las pirámides demográficas de 2011 (gráfico 3) dibuja dos escenarios para inicios de la década de 2010. El primer escenario lo constituye la capital, que incrementa su población como consecuencia del saldo migratorio. Ello provoca que, además, debido a la mayor natalidad de los inmigrantes extranjeros, aumenten suavemente las tasas de crecimiento vegetativo durante los últimos quince años. Bajo las cifras globales, se esconden dos procesos relevantes que otorgan un nuevo dinamismo a la demografía de la capital. Por un lado, se produce un gran contraste entre la población española, que se encuentra en proceso de envejecimiento y reducción, y la población extranjera, más joven, que utiliza la ciudad como plataforma de lanzamiento para movilidades subsecuentes. Por otro lado, bajo las pequeñas tasas de crecimiento, encontramos, como estudiaremos a continuación, un incremento muy sustancial de toda clase de movilidad hacia cualquier lugar (hacia dentro y hacia fuera, de la ciudad y de la región). Pueden trazarse, así, procesos significativos de recentralización urbana de la población y revitalización demográfica de algunos barrios (se registran hasta 200.000 cambios residenciales cada año en la ciudad de Madrid), junto a una reducción del atractivo de la capital en relación con su área metropolitana para determinados grupos sociales, tal y como ocurre en otros contextos metropolitanos españoles (Pujadas, 2009; López-Gay, 2014).

El segundo escenario es el configurado de manera mucho más compleja por el AM y la periferia. En este caso, la corriente principal que alimenta su crecimiento es la formada por la población activa no nativa y sus familias, como muestra el hecho de que la proporción de extranjeros se ensanche más por la base que en la capital. Dada la configuración histórica del territorio, buscan las localizaciones más baratas y bien conectadas del AM densa. Otras corrientes que podrían ayudar a configurar este territorio con una estructura demográfica más joven son la corriente de los nuevos hogares nacionales a la búsqueda de vivienda barata y estilos de vida suburbanos en el AM de baja densidad y la de una mezcla de población que llega a una periferia diversa, compuesta, por ejemplo, por viejos espacios recreativos convertidos en el hogar de nuevas familias, nuevos desarrollos urbanísticos, lugares colonizados a la búsqueda de estilos de vida más saludables, etc.

\section{Variaciones residenciales de nativos y no nativos durante la crisis económica}

Las tasas de desempleo constituyen una buena aproximación a la realidad de la crisis y de sus consecuencias socioeconómicas sobre la población. De hecho, 
Gráfico 3. Pirámides de población de la capital y del área metropolitana y la periferia
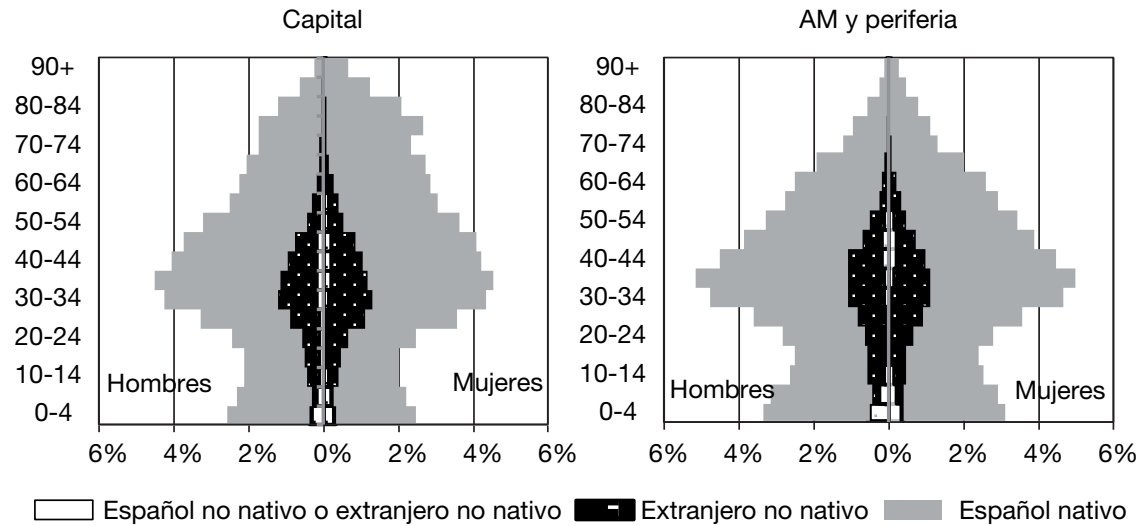

Fuente: Censo 2011. Elaboración propia.

alrededor del empleo y del desempleo, pueden modelarse muchas de las decisiones de movilidad en este contexto.

Atendiendo a los datos (gráfico 4), puede afirmarse que el contexto madrileño ha sido, en términos generales, mejor que el que presenta el conjunto del país, tanto en los años de bonanza como en los de crisis.

En todo el periodo, hay dos momentos dramáticamente intensos de crecimiento del desempleo. En el año 2008, las tasas del conjunto de España crecen rápidamente hasta situarse en torno al $18 \%$. Tras un pequeño valle (especialmente pronunciado en Madrid entre abril de 2010 y marzo de 2011), las cifras vuelven a subir fuertemente hasta alcanzar, en el primer trimestre de 2013, máximos de un $27 \%$ para España y de un $20 \%$ para Madrid. Es en este periodo cuando el hueco entre las tasas de España y de Madrid se ensancha, de modo que puede decirse que la posición relativa de la capital mejora claramente.

Las consecuencias de este escenario sobre la movilidad son fundamentalmente dos. Por una parte, el desempleo funciona como un determinante próximo de la movilidad hacia el exterior (López y Pérez, 2015) y como un factor de expulsión, lo que se encuentra indicado por el aumento de las emigraciones de no nativos (como veremos más adelante, en el gráfico 7). Por otra parte, la diferencia relativa de Madrid con el conjunto de España explica que los balances de movilidad que experimenta Madrid con el resto del país sigan siendo positivos, especialmente en el caso de los migrantes nativos.

Los indicadores de variaciones residenciales a lo largo del ciclo completo de auge y depresión muestran que, a partir del año 2000, la movilidad de nativos y no nativos se mantiene distribuida aproximadamente al cincuenta por ciento, si bien el tipo de movilidad que cada grupo realiza es bien diferente. 
Gráfico 4. Tasas de desempleo (población de 10 a 64 años) en Madrid y España, 2002-2015

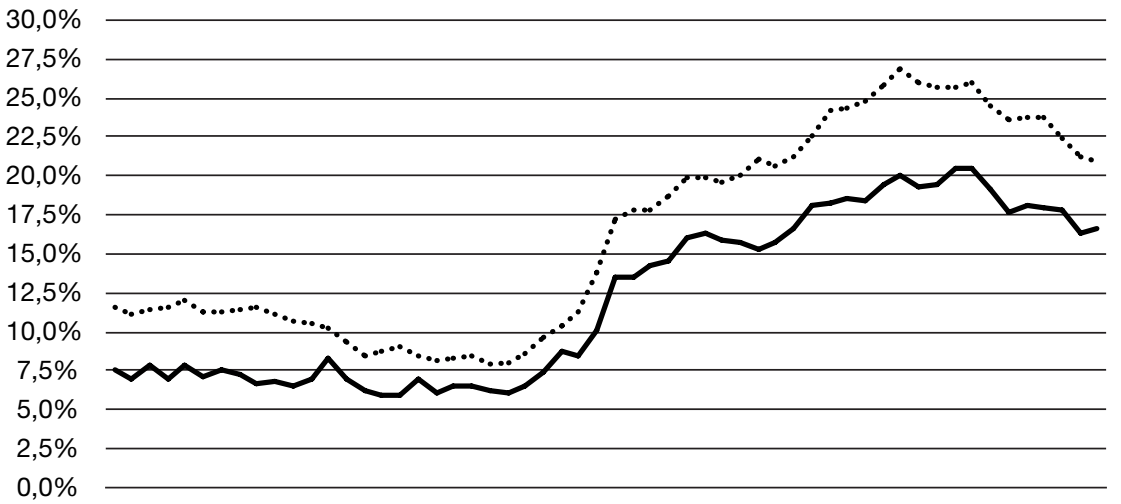

20022003200420052006200720082009201020112012201320142015

— C. Madrid $\quad \cdots . .$. España

Fuente: EPA. Elaboración propia.

Los no nativos se concentran en las migraciones internacionales, mientras que los nativos lo hacen en las migraciones internas y en las regionales (gráfico 5).

Una de las tendencias más claras que conecta con la idea de que nos hallamos en una era de la movilidad es que esta ha llegado para quedarse. Durante el periodo 1999-2007, la movilidad residencial entre municipios se triplicó. E incluso en tiempos de crisis, las cifras más que doblan las del año 2000. La población no nativa ha desempeñado un papel muy importante en el aumento de la movilidad, pero la población nativa también ha incrementado significativamente su contribución (de los 180.000 movimientos del año 2000 a los 280.000 de 2015).

La recesión produjo una reducción instantánea de la movilidad residencial (en 2008 y en 2009), pero, a la postre, la cifra media de movimientos es mucho más alta que antes de la crisis. Hay también cierta evidencia de que, en la fase inicial de la crisis, mientras más duro era el contexto, mayor cautela había para emprender movimientos. Las decisiones de movilidad residencial o migración necesitan tiempo hasta que se llevan a cabo, y las variaciones en el ciclo económico (como el pequeño respiro entre 2010 y 2011, seguido del duro repunte del desempleo) podrían actuar como factores próximos de la decisión.

Para comprender los procesos de transformación de la movilidad residencial durante la crisis, resulta necesario entender el comportamiento diferenciado de los no nativos y los nativos. La recesión produjo una reducción instantánea de la movilidad de los no nativos, que parecían esperar coyunturas específicas, como el año 2011, en el que la crisis volvió a arreciar tras un periodo menos duro, mientras que la de los nativos no registró cambios tan significativos. 
Gráfico 5. Variaciones residenciales en la Comunidad de Madrid, según alcance del movimiento y origen de la persona, 1997-2015

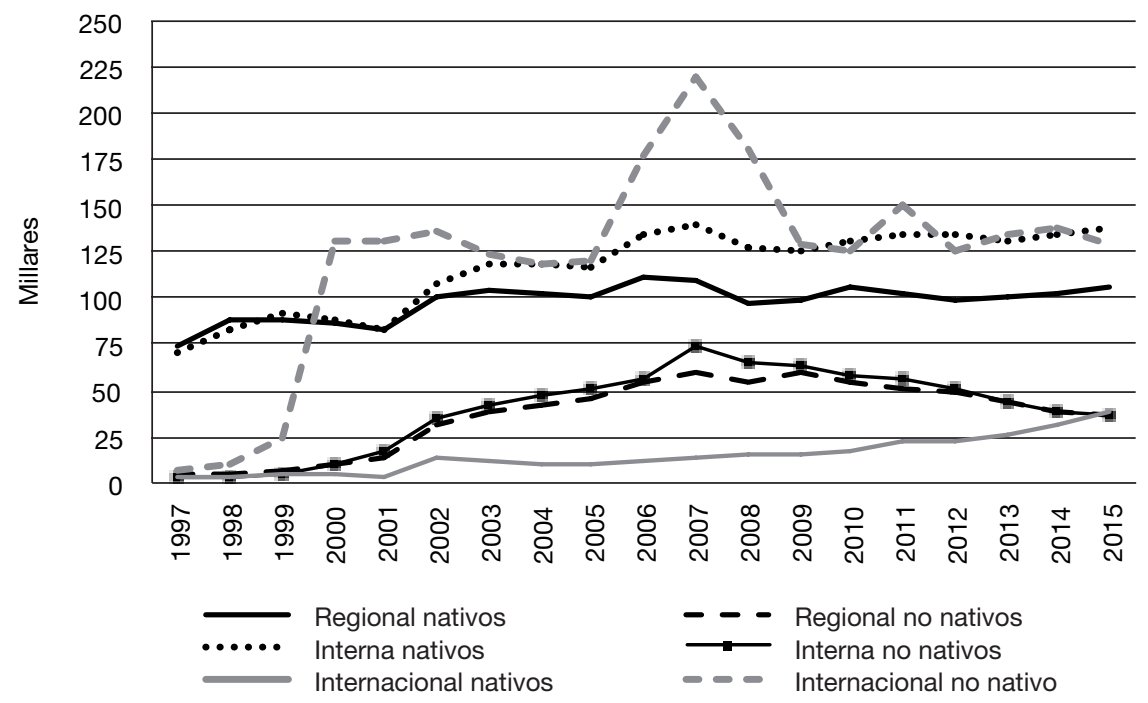

Fuente: EPA. Elaboración propia.

El aumento general de la movilidad determina cierta dinamización del territorio. Sin embargo, una evaluación más precisa requiere atender al sentido y a la dirección de estos movimientos. Una primera aproximación consiste en observar el peso de la movilidad de nativos en cada uno de los movimientos (regionales, migraciones internas y migraciones internacionales) (gráfico 6).

En el periodo 1997-2007, la movilidad interna y las emigraciones estaban dominadas por los nativos, aunque cada vez más los no nativos participaban en estos movimientos. Las inmigraciones internacionales, por su parte, eran el campo claramente mayoritario de los no nativos, que, entre 2000 y 2008 , supusieron en torno a un $95 \%$ de las inmigraciones anuales y fue el flujo dominante entre todos los analizados.

Desde 2007, la distribución por origen de los flujos de movilidad cambió. Por una parte, las inmigraciones revirtieron su tendencia, en la medida en que los no nativos redujeron su participación en estos flujos. Por otra parte, a la vez que se reducían las inmigraciones internacionales de los no nativos, aminoraban sus salidas, en un movimiento muy significativo en relación con la crisis económica. Finalmente, el propio peso de los no nativos en la movilidad regional (que llegó a ser de casi el $40 \%$ ) se redujo hasta menos del 30\%.

Las estructuras de movilidad de nativos y no nativos durante la crisis muestran algunas características muy útiles para comprender lo que ha ocurrido (gráfico 7). En conjunto, los movimientos de los no nativos estaban dominados 
Gráfico 6. Porcentaje de migrantes nativos, según tipo de movimiento. Comunidad de Madrid, 1997-2015

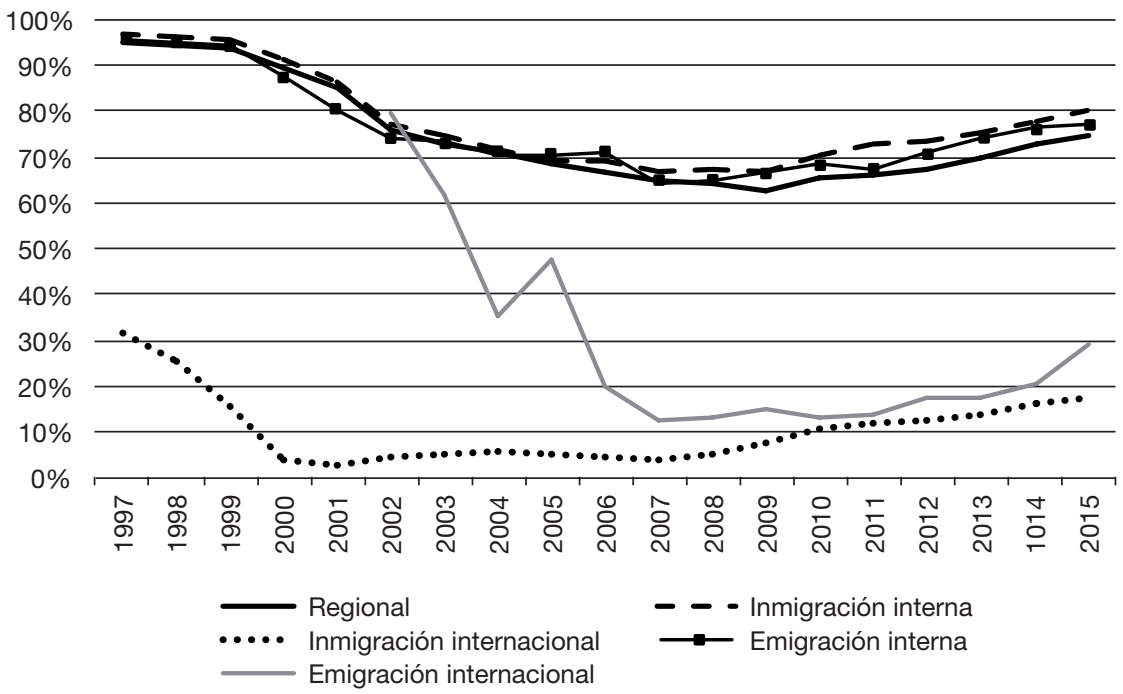

Fuente: EVR. Elaboración propia.

por una fortísima inmigración internacional. La recesión produjo una rápida caída de las inmigraciones internacionales y, en seguida, un aumento moderado de las emigraciones internacionales, lo que llevó en poco tiempo a un volteo de los saldos migratorios de no nativos en la Comunidad de Madrid. Desde 2010, hay más no nativos saliendo al extranjero desde la región que entrando en ella. Por otra parte, existe también una moderada tendencia a reducir el número de migraciones internas de no nativos. En este caso, los saldos resultan positivos durante la crisis. Por su parte, la movilidad regional de los no nativos va reduciéndose poco a poco.

El ejemplo de la movilidad nativa es bien diferente. En este caso, la movilidad regional y las migraciones internas destacan sobre las migraciones internacionales. Además, la incidencia global de la crisis sobre la movilidad residencial de los nativos ha sido significativamente diferente. De este modo, durante este periodo, la movilidad regional continúa siendo el flujo más relevante. La cuestión más interesante es el cambio en el saldo de migraciones internas de los nativos, que se produce a partir del año 2010. Al contrario que el saldo de los no nativos, dominado por el cambio en el régimen de las migraciones internacionales, en ese año, comienzan a entrar más nativos de los que salen de la Comunidad, gracias al saldo positivo de las migraciones internas. La posición relativamente mejor de Madrid frente al contexto nacional durante la crisis podría explicar cierto atractivo para los nativos que deciden migrar desde otras regiones. 
Gráfico 7. Movilidad residencial según tipo de movimiento. Nativos y no nativos. Comunidad de Madrid, 2007-2015
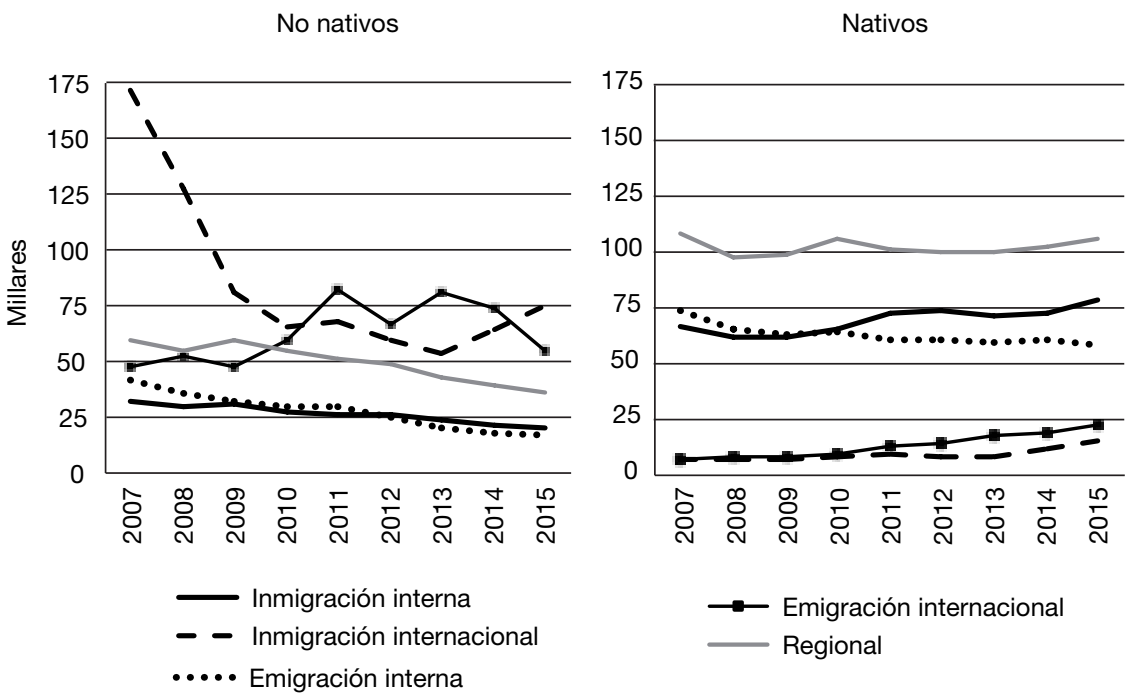

Fuente: EVR. Elaboración propia.

\section{Movilidad y reestructuración territorial de la región madrileña}

Las tendencias de movilidad residencial observadas tienen consecuencias sobre el territorio. De un modo estático, podemos comprobar sus resultados sobre el aumento y la composición de la población en cada área. Si observamos el detalle de las tasas de crecimiento entre 2007 y 2015, en el gráfico 8, observamos que existe una especie de reacción territorial en cadena a la crisis, de manera que se reducen las cifras de crecimiento, primero, en la capital, luego, en el AM y, finalmente, en la periferia. De manera hipotética, podríamos decir que la capital funciona como puerta de entrada a los nuevos inmigrantes provenientes de otras regiones y del extranjero. En la medida en que las condiciones económicas cambian, crece la emigración de los no nativos desde la Comunidad de Madrid hacia otros países. La función de distribución que ejercía Madrid colapsa, lo que reduce la movilidad regional de los no nativos hacia el AM y la periferia. En el AM, las poblaciones no nativas, con un grado mayor de asentamiento, se toman más tiempo para decidirse a realizar nuevos movimientos residenciales. Por último, en la periferia, pese a que su crecimiento migratorio es una tendencia con poco peso dentro del conjunto, este resulta más independiente de la llegada de pobladores no nativos. De este modo, aunque finalmente sí se observa un claro efecto de la crisis, los efectos son, en general, más tardíos $\mathrm{y}$, sobre todo, más pequeños.

El crecimiento natural resulta, como ya hemos dicho, ligado indirectamente a la movilidad de las poblaciones más jóvenes y no nativas. Eso ayuda a expli- 
Gráfico 8. Tasas por mil de crecimiento anual de la población por área, 2007-2015
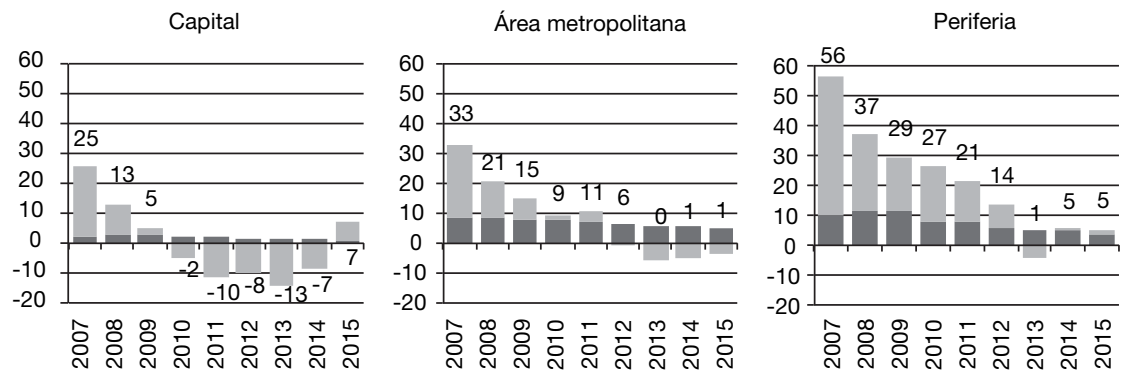

Tasa de crecimiento migratorio

Tasa de crecimiento vegetativo

Fuente: Padrón y MNP. Elaboración propia. La cifra sobre cada barra indica el crecimiento total.

car, junto a la tendencia hacia el envejecimiento de la población, que las tasas de crecimiento vegetativo se reduzcan una vez que se ha producido la caída del crecimiento migratorio.

En términos generales, Madrid ha sido un escenario fundamental para entender la llegada masiva de inmigrantes internacionales a España durante el nuevo siglo, puesto que ha alcanzado globalmente tasas muy superiores a las del conjunto de España. No obstante, este impacto no ha sido igual en todo el territorio. Según las cifras del Padrón, de las tres áreas analizadas dentro de la Comunidad de Madrid, la capital ha albergado las concentraciones mayores de no nativos (llegó a un 21\% en 2010). Tanto el AM como la periferia, habían experimentado concentraciones algo menores durante el periodo anterior a la crisis y, sobre todo, su evolución ha estado relacionada con la llegada de contingentes a la capital, a partir de donde se distribuían. El AM constituye, de hecho, el escenario donde los no nativos han logrado un mayor asentamiento. Durante el periodo de crisis, la inversión de las tasas migratorias redujo la concentración de no nativos en todos los escenarios a partir de 2010.

Una vez mostrada la importancia cuantitativa de la movilidad residencial en los balances territoriales, la significatividad de los movimientos de la población no nativa y la manera en que la crisis ha frenado algunas tendencias, cuando no las ha revertido, nos queda por ver de qué modo ha influido la movilidad registrada durante el ciclo completo de expansión y crisis en los procesos de configuración del territorio. Tal y como se explica en el apartado metodológico, hemos distinguido entre movimientos centralizadores, metropolizadores, desconcentradores e internos. Para conseguir un cuadro completo, se incluyen aparte las tasas migratorias que obtiene cada escenario, tanto a nivel doméstico como internacional. De este modo, el gráfico 9 puede contemplarse como un plano de las trayectorias seguidas por los diversos procesos que conforman la región durante este periodo. Su lectura es compleja, pero, a través de los seis procesos siguientes, nos ofrece algunas indicaciones claras de lo ocurrido. En general, debe tomarse en cuenta que la profundidad de los procesos depende 
Gráfico 9. Tasas de movilidad regional (9A), migratoria interna y migratoria internacional (9B) por mil habitantes, según el tipo de movilidad (Comunidad de Madrid, 1997-2015)

(9A) Movilidad regional

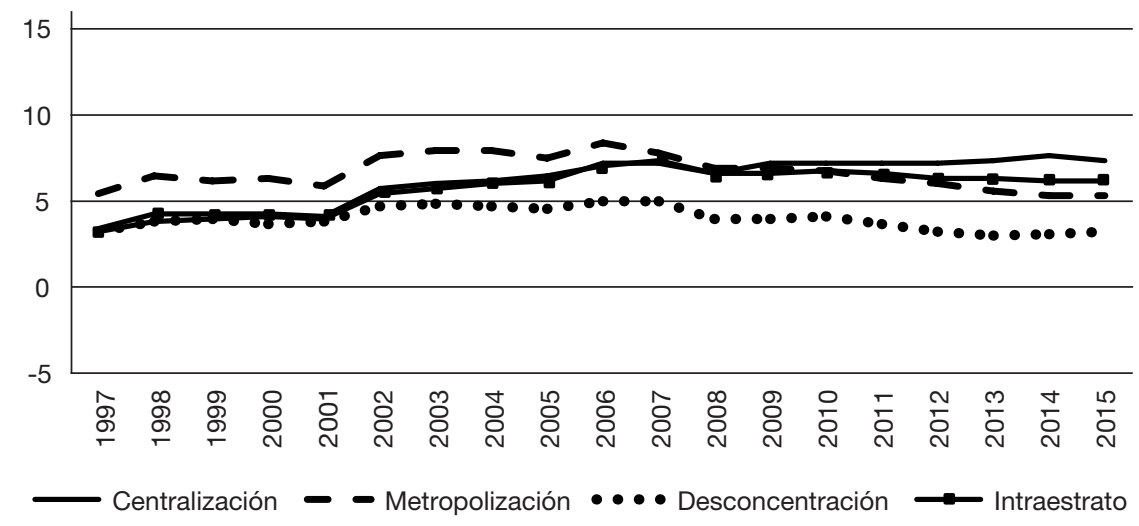

(9B) Migraciones internas (en negro) e internacionales (en gris)

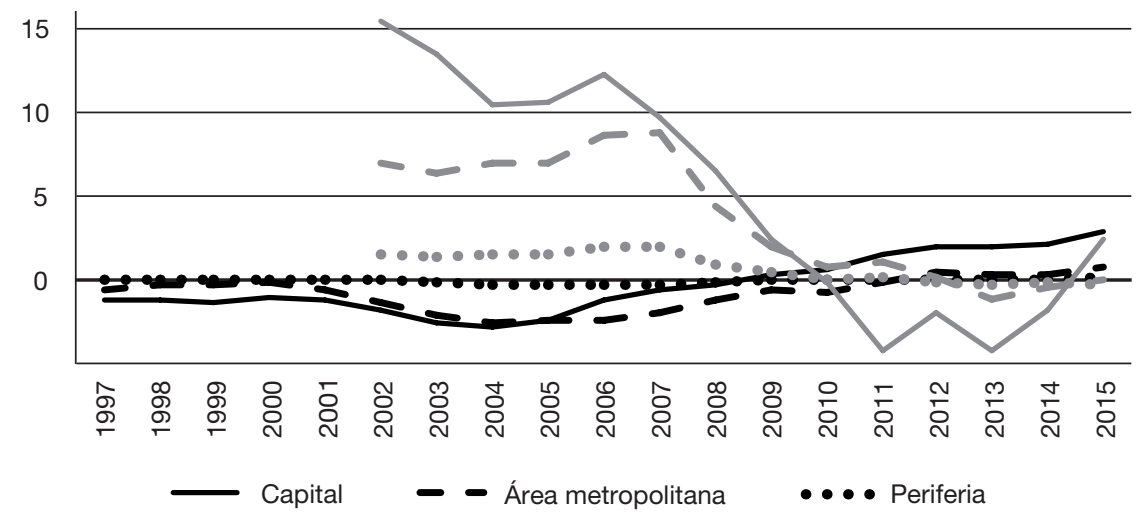

Fuente: EVR y Padrón. Elaboración propia.

de los volúmenes de población implicados en las tasas para cada año y que los procesos de movilidad regional de la Comunidad de Madrid son apoyados o revertidos por las tasas migratorias con el exterior de la región.

En primer lugar, las tasas de movilidad migratoria internacional (línea continua gris) vienen a apuntalar la hipótesis de que ha existido una falsa centralización (Galiana y Vinuesa, 2012): la capital constituye el punto de entrada de los flujos internacionales de migrantes que no solo serán distribuidos por la región, sino también a través de todo el país. De hecho, el crecimiento migratorio de la capital es predominante únicamente en los primeros años, mientras que, a partir de 2002, los procesos de movilidad residencial regional (gráfico 
9A) van tomando protagonismo. De este modo, parte de los efectos sobre la población de la capital son intrínsecamente temporales.

En segundo lugar, se observa una redistribución demográfica: el ascenso de la movilidad regional junto al fuerte declive del crecimiento migratorio de la capital (que recoge la ola de entrada procedente de fuera, especialmente del extranjero) indica que, de acuerdo con el análisis de Pozo y García-Palomares (2011), una vez que los recién llegados se convierten en residentes permanentes, buscan nuevas localizaciones adaptadas a sus expectativas, generalmente fuera de la capital. Ello hace aumentar todas las tasas de movilidad regional. Singularmente, las cifras de movimientos metropolizadores (línea discontinua en el gráfico 9A). En el gráfico 10B, se muestra cómo las tasas de movilidad regional de los no nativos se frenan con la crisis.

En tercer lugar, aunque no es materia particular de este trabajo, las cifras de población y empleo, así como los indicadores de movilidad residencial y commuting, desagregados por las diferentes zonas que componen el área metropolitana, indican un doble proceso en el AM, que entronca con las diferencias regionales entre el AM densa del este y el sur y el AM dispersa del norte y el oeste (Leal, 2004). El gráfico 9 muestra que las tasas de movilidad metropolizadora (9A) y la tasa de movilidad migratoria interna (9B) del AM (línea discontinua), que venían menguando en los años noventa debido a cierta colmatación de los espacios densos mayoritarios (y, por lo tanto, con mayor peso en las cifras totales del AM), repuntan con la llegada de la inmigración extranjera. A causa de la crisis, el efecto metropolizador del asentamiento de extranjeros va diluyéndose, con lo que se vuelve a registrar una reducción de la movilidad metropolizadora. Este proceso general de agotamiento de la metropolización densa convive, no obstante, con la tendencia, ya observada en la década de los noventa, hacia una metropolización difusa fluida. Si bien este no es el objeto del presente trabajo, apuntaremos que, en las zonas norte y oeste, se ha seguido creciendo en términos de empleo y residencia durante la crisis, con lo que se han afianzado como unos de los espacios más dinámicos de la región.

Un cuarto proceso lo constituye lo que denominamos centralización adaptativa. Durante la crisis, muchos movimientos regionales y migraciones internas se han dirigido hacia el centro (línea continua), sobre todo porque la aglomeración urbana albergaba el mercado de trabajo más dinámico y resistente, además de reducir algunos de los inconvenientes de la vida en las periferias, como las distancias o la falta de servicios. Aunque la capital perdió población durante la recesión debido a la fortísima corriente de salida hacia otras áreas de la región o de España y hacia el extranjero, lo cierto es que la centralización se ha convertido en el primer vector de movilidad residencial interna en la Comunidad durante la crisis económica.

En relación con el policentrismo, en quinto lugar, utilizamos como indicador la movilidad regional de los estratos (trazo continuo con marcador en el gráfico 9A), la mayor parte de la cual representa cambios de residencia entre municipios dentro del AM. A pesar de que Madrid es la cabeza indiscutible del sistema urbano regional, un conjunto de ciudades medias bien conectadas 
(especialmente en el AM) ha hecho posible la constitución de un conjunto de nuevos flujos internos muy relevantes, de acuerdo con las investigaciones de Roca et al. (2011), Gallo et al. (2010) y Gallo y Garrido (2012).

Nos referimos, en sexto y último lugar, a la existencia de una sólida corriente de débil desconcentración hacia la periferia regional. Esta corriente se reduce un poco durante el periodo de crisis, sin llegar a desaparecer. Lo más notable es precisamente su fortaleza durante más de veinte años. Aunque su volumen y su intensidad no son muy relevantes, su solidez y una cierta insensibilidad hacia las coyunturas la convierten en una corriente claramente diferenciada, en la que se dan procesos complejos y diversos como la ocupación urbana de los bordes metropolitanos, las promociones urbanísticas en pueblos bien conectados, nuevas residencias rurales, etc. Durante la fase de auge económico, la movilidad regional tiende a nutrir estas áreas, mientras que dicho proceso se reduce en la fase de crisis. Por el contrario, las migraciones internas, en un volumen mucho más modesto, señalan la tendencia inversa. Los saldos negativos de los tiempos de auge comienzan a revertirse con la crisis.

El estudio de las corrientes de movilidad según el origen de sus protagonistas, reflejado en el gráfico 10, refuerza las evidencias sobre algunos de los procesos señalados. Debe observarse, en primer lugar, que la escala de las tasas de movilidad para ambos grupos es diferente. Los no nativos tienden a moverse mucho más y en casi todas las direcciones, como consecuencia del anclaje menor que tienen en la sociedad en la que residen. Así, carecen muchas veces de redes familiares extensas, sus vínculos identitarios se encuentran desterritorializados y no han contraído obligaciones hipotecarias en mayor medida que los nativos. Sin embargo, al margen de la evidente diferencia cuantitativa, nos interesa destacar el hecho de que las pautas de movilidad en la región tienden a converger a lo largo de la crisis.

En relación con las migraciones internacionales, las tasas se encuentran claramente vinculadas al episodio de la ola migratoria internacional iniciada a finales de los años noventa. Esta migración masiva causa efectos temporales, como hemos visto, pero también estructurales. Los efectos temporales de la ola inmigratoria son, por ejemplo, los ya mencionados de centralización y redistribución demográfica, significativamente a través de movimientos metropolizadores, pero también a través de toda la región urbana (policentrismo) e incluso de la periferia.

Con la llegada de la crisis económica, muchos extranjeros fueron desplazados fuera del mercado laboral. Una parte importante de ellos optaron por emigrar a sus países de origen, lo que se observa en la inversión de las tasas migratorias con el exterior de los no nativos (10A). Por el contrario, para los españoles (línea continua negra en 10B y 10C), Madrid, especialmente la capital, conserva cierto atractivo durante la crisis, en parte explicable por la posición de ventaja de Madrid en relación con el conjunto de España, como hemos visto al analizar la evolución del desempleo.

Sin embargo, una vez resistido el primer embate de la crisis, aquellos no nativos que permanecen, presumiblemente mejor establecidos, adoptan 
Gráfico 10. Tasas de movilidad regional, migratoria interna y migratoria internacional por mil habitantes, según tipo de movilidad (nativos/no nativos), 2007-2015

(10A) Migraciones internacionales
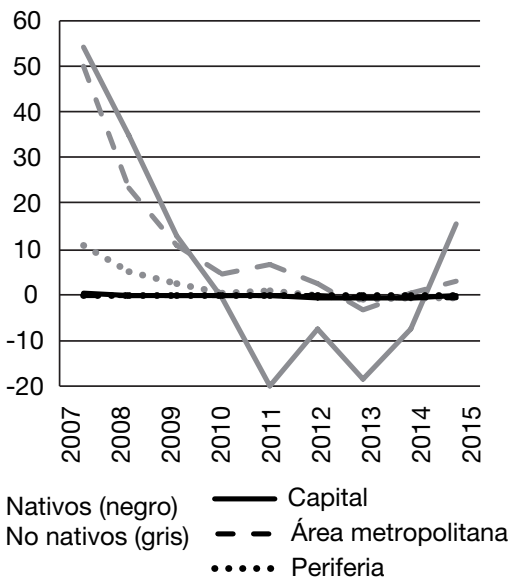

(10C) Migraciones internas (nativos)

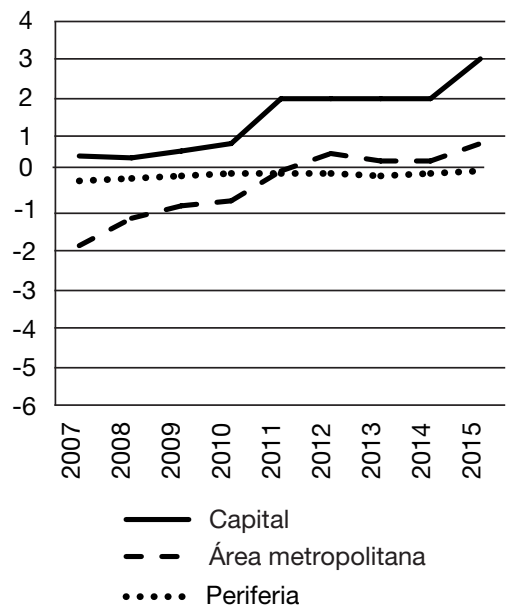

(10B) Movilidad regional

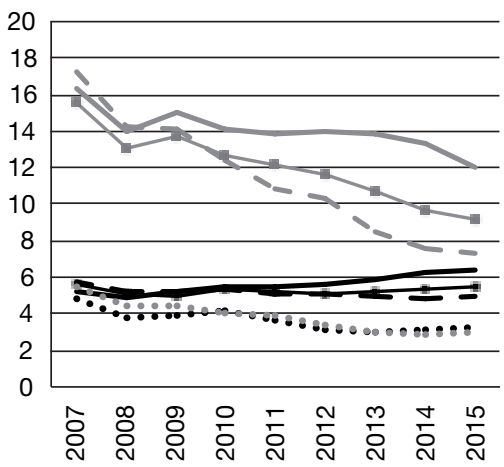

—Centralización …. Desconcentración

— - Metropolización _ـ Interestrato

(10D) Migraciones internas (no nativos)

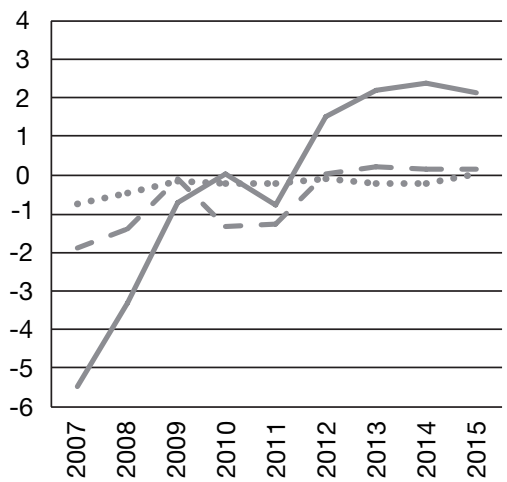

Fuente: EVR y Padrón. Elaboración propia.

corrientes de movilidad residencial que tienden a converger con las de los nativos. De este modo, una vez disminuido el efecto coyuntural de las migraciones internacionales, los desplazamientos de los no nativos comienzan a cobrar más importancia como parte de las corrientes de movilidad regional y de las migraciones internas dominadas por los nativos. La movilidad regional de los no nativos (10B) se reduce significativamente y adopta una estructura similar a la de los nativos, con un predominio de los movimientos centralizadores sobre el resto. En términos dinámicos, la crisis ha visto crecer la movilidad regional 
centralizadora y el crecimiento migratorio de la capital. Por su parte, el proceso de asentamiento, típicamente indicado por la metropolización, se reduce (líneas discontinuas en 10B). Paradójicamente, el aumento del crecimiento migratorio interno del AM durante la crisis (línea discontinua en 10C y 10D), especialmente por los nativos, pero también, finalmente, por los no nativos, indica, al igual que los movimientos regionales desde la periferia (incluidos en las tasas de centralización), un aumento de la centralización en las dinámicas de movilidad. Así pues, estos movimientos migratorios, aunque con efectos muy modestos, siguen la estela de las migraciones típicamente centralizadoras dirigidas hacia la capital (línea continua en 10C y 10D).

\section{Conclusión}

Los procesos de reestructuración espacial en la era de las movilidades se encuentran crecientemente relacionados con los flujos globales, así como con las estrategias locales de movilidad de carácter adaptativo.

Madrid es una región fuertemente integrada y encabezada por un municipio muy fuerte demográficamente, que todavía ejerce una gran dominación funcional sobre el territorio.

El proceso de modernización configuró tres áreas (la capital, el AM y la periferia). Sus balances internos han ido conformando el territorio a lo largo del eje formado por la concentración y la desconcentración. Entre 1960 y 1980, a través de un proceso de centralización y metropolización densa. Entre 1980 y 2000, mediante una metropolización de baja densidad y cierta contraurbanización. Entre 2000 y 2015, encontramos un nuevo escenario como producto de la ola inmigratoria y la gran crisis. En este contexto, la movilidad crece en todas las direcciones y en todos los sentidos.

Los migrantes internacionales han sido un factor fundamental en la reorganización del territorio durante los últimos quince años. No es que se hayan convertido solo en una parte importante de las cifras de población generales, sino que han realizado contribuciones clave a asuntos demográficos trascendentales como el de la sostenibilidad de los escenarios estudiados en el medio plazo. Las migraciones internacionales han ido acompañadas por un incremento general de toda clase de movilidad, incluida la regional y las migraciones internas de los nativos. El volumen y la rapidez de las migraciones internacionales han provocado que algunos de los efectos sean meramente temporales, como es el caso de la falsa centralización en la capital o la consecuencia que comporta: la redistribución por el territorio de los recién llegados a la capital. Sin embargo, se observa finalmente una convergencia entre las movilidades de los nativos y los no nativos. El marco de la globalización y la movilidad adaptativa puede aplicarse adecuadamente a los efectos sobre la reorganización del territorio.

Durante la crisis, el estudio de la movilidad muestra, en primer lugar, una fortísima reducción de los inmigrantes internacionales, un aumento de la emigración no nativa y una caída de los efectos temporales asociados a la ola inmigratoria. En segundo lugar, puede afirmarse, no obstante, que la movilidad ha venido 
para quedarse. La reducción en los volúmenes totales de movilidad es pequeña si la comparamos con la profundidad de la crisis. La movilidad regional y las migraciones se han convertido, por lo tanto, en un recurso central para encarar la situación. Por último, la convergencia entre las estrategias de nacionales y extranjeros muestra la adecuación del marco analítico utilizado. Si descontamos el efecto de los balances migratorios internacionales, el proceso que más llama la atención es el reforzamiento de la movilidad regional y la migración interna con efectos centralizadores, especialmente en el caso de los nativos, mientras que las movilidades metropolizadoras disminuyen, si bien esta es una cuestión que requiere una inspección más precisa dada la conocida dualidad del AM.

\section{Referencias bibliográficas}

BAYONA-I-CARRASCO, J. y PUJADAS-I-RÚBIES, I. (2014). «Movilidad residencial y redistribución de la población metropolitana: Los casos de Madrid y Barcelona». EURE [en línea], 40 (119), 261-287. <https://doi.org/10.4067/s0250-71612014000100012>.

CASTELLS, M. (1997). La era de la información: Economía, sociedad y cultura. Volumen 1. La sociedad red. Madrid: Alianza.

FERIA, J.M. (2008). «Un ensayo metodológico de definición de las áreas metropolitanas en España a partir de la variable residencia-trabajo». Investigaciones Geográficas [en línea], 46, 49-68. <https://doi.org/10.14198/ingeo2008.46.03>.

FOLKE, C. (2006). «Resilience: The emergence of a perspective for social-ecological systems analyses». Global Environmental Change [en línea], 16, 253-267. $<$ https://doi.org/10.1016/j.gloenvcha.2006.04.002>.

GalianA, L. y VinUeSA, J. (2012). «Descentralización y recentralización en espacios metropolitanos maduros: El caso de Madrid». En: VArelA, B. y VinUeSA, J. (coord.). Metrópolis: Dinámicas Urbanas. Madrid: Universidad Autónoma.

GALLO, M.T. y GARRIDO, R. (2012). «Una aproximación a la estructura urbana policéntrica en la Comunidad de Madrid». ACE, 18, 69-100.

Gallo, M.T.; Garrido, R. y Vivar, M. (2010). «Cambios territoriales en la Comunidad de Madrid: Policentrismo y dispersión». EURE [en línea], 36 (107). <https://doi.org/10.4067/s0250-71612010000100001>.

Giddens, A. (1985). «Time, Space and Regionalization». En: Social Relations and Spatial Structures [en línea]. Londres: Macmillan Education, 265-295. <https://doi.org/10.1007/978-1-349-27935-7_12>.

Gil-Alonso, F.; BAYONA-I-CARRASCO, J. y PUJADAS-I-RÚBIES, I. (2013). «From boom to crash: Spanish urban areas in a decade of change (2001-2011)». European Urban and Regional Studies [en línea], 3 (2). <https://doi.org/10.1177/0969776413498762>.

- (2015). «Las migraciones internas de los extranjeros en España: Dinámicas espaciales recientes bajo el impacto de la crisis». Boletín de la Asociación de Geógrafos Españoles, 69, 233-261.

Gutiérrez-Puebla, J. y García-Palomares, J.C. (2007). «New spatial patterns of mobility within the metropolitan area of Madrid: Towards more complex and dispersed flow networks». Journal of Transport Geography [en línea], 15 (1), 18-30. $<$ https://doi.org/10.1016/j.jtrangeo.2006.01.002>. 
Harvey, D. (1989). The condition of posmodernity. Cambridge y Oxford: Blackwell.

Held, D.; McGrew, A.; Goldblatt, D. y Perraton, J. (1999). Global Transformations: Politics, Economics and Culture. Cambridge: Polity Press.

Holling, C. (2001). «Understanding the Complexity of Economic, Ecological, and Social Systems». Ecosystems [en línea], 4, 309-405. <https://doi.org/10.1007/s10021-001-0101-5>.

Kaufmann, V.; Bergman, M. y Joye, D. (2004). "Motility: mobility as capital». International Journal of Urban and Regional Research [en línea], 28 (4). <https://doi.org/10.1111/j.0309-1317.2004.00549.x>.

LEAL, J. (2004) «Segregation and social change in Madrid metropolitan region». The Greek Review of Social Research [en línea], 113A, 81-104. <https://doi.org/10.12681/grsr.9219>.

LOCKE, C.; AdGER, W.N. y KeLly, P.M. (2000). «Changing places: Migration's social and environmental consequences». Environment: Science and Policy for Sustainable Development [en línea], 42 (7), 24-35. <https://doi.org/10.1080/00139150009605748>.

López de Lera, D. y PÉrez, A. (2015). «La decisión de retornar en tiempos de crisis: Una perspectiva comparada de los migrantes ecuatorianos y rumanos en España». Migraciones [en línea], 37, 171-194. <https://doi.org/10.14422/mig.i37.y2015.008>.

LOPEZ-GAY, Antonio (2014). «Population growth and re-urbanization in Spanish inner cities: The role of internal migration and residential mobility». Revue Quetelet I Quetelet Journal [en línea], 1 (2), 67-92. <https://doi.org/10.14428/rqj2013.01.02.03>.

MódenEs, J.A. (2007). «Una visión demográfica de la movilidad residencial reciente en España». En: FERIA, J.M. (coord.). La vivienda y el espacio residencial en las áreas metropolitanas. Sevilla: Centro de Estudios Andaluces.

Monzón, A. y Hoz, D. de la (2009). «Efectos sobre la movilidad dinámica territorial de Madrid». Urban, 14, 58-71.

Moreno Jiménez, A. (1999). «La distribución espacial de la población en la Comunidad de Madrid». En: Población y espacio en la Comunidad de Madrid: Análisis y aplicaciones a nivel microgeográfico. Madrid: Comunidad de Madrid.

Newland, K. (2009). Circular Migration and Human Development. Human Development Research Paper Series, Vol. 42. United Nations Development Programme.

Pino ARTACHO, J.A. del (2001). «Hacia la comprensión del espacio urbano global: ¿Sistemas de ciudades o redes urbanas». Espacio, Tiempo y Forma [en línea], 14. <https://doi.org/10.5944/etfvi.14.2001.2587>.

- (2013). «Dinámica territorial en la era de la movilidad». En: LuCAS, A.; CORTÉs, C. y CÁCERES, M.D. Madrid en el siglo XXI: Transformaciones y retos de su realidad social. Madrid: Fragua.

- (2015). Estructuras residenciales y movilidad: Más allá de la segunda residencia. Madrid: CIS.

Pozo Rivera, E. y García-Palomares, J.C. (2011). «Evolución reciente y pautas de distribución espacial de las migraciones internas de extranjeros: El caso de la Comunidad de Madrid (1997-2008). Scripta Nova: Revista Electrónica de Geografía y Ciencias Sociales, XV (384).

PUJADAS-I-RÚBIES, I. (2009). «Movilidad residencial y expansión urbana en la Región Metropolitana de Barcelona, 1982-2005». Scripta Nova: Revista Electrónica de Geografia y Ciencias Sociales, XIII (290). 
RECAÑO, J. (2016). «La consolidación de las migraciones internas de inmigrantes como factor estructural de la movilidad geográfica en España». Panorama Social, 24, 49-71.

Roca, J.; Arellano, B. y MoIX, M. (2011). «Estructura urbana, policentrismo y "sprawl": Los ejemplos de Madrid y Barcelona». Ciudad y Territorio, 43, 168-299.

SAlCEDO, J. (1977). Madrid culpable: Sobre el espacio y la población en las ciencias sociales. Madrid: Tecnos.

Sassen, S. (2000). Cities in a World Economy. 2. ${ }^{\mathrm{a}}$ ed. Thousand Oaks: Pine Forge Press.

Sheller, M. y Urry, J. (2016). «Mobilizing the new mobilities paradigm». Applied Mobilities [en línea], 1 (1), 10-25.

<https://doi.org/10.1080/23800127.2016.1151216>.

URRY, J. (2000). Sociology beyond societies. Londres: Routledge.

VAlenzuela, M. (2011). «Los procesos de metropolización: Madrid, una región metropolitana entre la dispersión y el policentrismo». En: España en la Unión Europea: Un cuarto de siglo de mutaciones territoriales, 211-253. Colección de la Casa de Velázquez, 121.

VinUESA, J. (2004). «Un nuevo modelo migratorio». En: Características demográficas de la Comunidad de Madrid según el Censo de 2001. Madrid: Comunidad de Madrid.

- (2016). «La movilidad interna e internacional de los inmigrantes rumanos durante la crisis». Scripta Nova: Revista Electrónica de Geografía y Ciencias Sociales, XX (536).

Waldinger, R. (2013). «Immigrant transnationalism». Current Sociology [en línea], 61 (5-6), 756-777.

<https://doi.org/10.1177/0011392113498692>. 